\title{
Morphological sharpening of color images
}

\author{
S. SKONECZNY* \\ Institute of Control and Industrial Electronics, Warsaw University of Technology, 75 Koszykowa St., 00-662 Warsaw, Poland
}

\begin{abstract}
This paper presents a novel approach to morphological contrast sharpening of image using the multilevel toggle operator. The concept presented here is a generalization of toggle based contrast operator for gray-level images. The multilevel toggle operator is used to enhance the contrast of multivalued images. In order to perform necessary morphological operations the modified pairwise ordering (MPO) algorithm is proposed. It gives the total order of color pixels. For comparison four other ordering methods are used. The main advantage of the proposed sharpener is its significant contrast enhancing ability when using MPO. Theoretical considerations as well as practical results are shown. Experimental results show its applicability to low-contrast color images.
\end{abstract}

Key words: color image sharpening, multilevel toggle, mathematical morphology.

\section{Introduction}

Contrast for monochromatic images is most often defined as a measure of the variation in intensity of pixels. Analogically for color images it is possible to formulate a new definition of variation in at least one of three components: luminance (intensity), hue or saturation. The area of these variation is usually a small window, and in such a case the concept of contrast is localized. We can formulate, however, another measure concerning the whole image or at least majority of its pixels which leads to the notion of global contrast. In normal circumstances, however for improving the appearance of color image the local contrast seems to be a little bit more advantageous that its global counterpart.

There are lots of pictures suffering from low contrast phenomenon. In many cases this fact reduces the visual quality of an image which is shown to a human viewer. Sometimes the image quality is completely unacceptable, in particular, in pattern recognition applications. There are many examples of such defocused pictures, especially in the area of old pictures which are important for the cultural heritage of human beings [1]: like artistic wall-paintings (e.g. old church paintings) or frames taken from old motion pictures etc. In particular for medical images the increasing of contrast is a very important factor and it helps significantly in a segmentation process [2], as well as in motion tracking using optical flow in 3D image research [3]. The aim of this paper is to generalize the toggle based gray-level contrast operator to multilevel toggle sharpener for enhancing the contrast of color images. The paper is organized as follows. In Sec. 2 the state of art is presented. The most important Sec. 3 presents the novel multistage toggle contrast operator. In Sec. 4 the Classical as well as Modified Pairwise Ordering (MPO) are described. Other ordering schemes are given in Sec. 5. Contrast measure expression for color images is described in Sec. 6. Sections 7 and 8 include respectively experiments and final conclusions. Section 9 presents the proof that the MPO is the total order.

\section{Morphological contrast enhancement - state of the art}

There have been some attempts to enhance the image by increasing its contrast using the tools that offer mathematical morphology. One of the main improvements of image contrasts in the area of mathematical morphology takes advantage of notion of toggle mappings. The fundamental concept of utilizing the toggle mappings is to compare all pixels in the original image with a set of patterns and then to select the nearest value, in the sense of some previously defined distance, from the patterns with respect to the original image. The original idea stems from a paper of Kramer and Bruckner [4]. They took a dilation and an erosion, both working with flat structuring elements, as the set of two patterns or, in another words, two primitives. Their method requires to choose one extensive and the other anti-extensive primitive to construct toggle mapping, which may lead to instability problems when this transformation is used iteratively. This means the degradation of a processed image [5]. Meyer and Serra [6] developed the theory of contrast mappings and they used morphological idempotent transformations that might be useful for contrast enhancement. In their paper they demonstrate two-state and three-state contrast operators for gray level images. On the other hand, Serra in [5] demonstrated two examples of idempotent toggle mappings using two and four primitives respectively. The theory of toggle-like mappings is nowadays still being developed and applied to image processing - a good example is the conditional toggle mapping [7].

Angulo [8], by the analogy to the two-state contrast operator for gray-scale imagery, proposed a color contrast mapping, with ordering based on pixel distances to reference color. The three-state toggle based contrast operator for color images was suggested by Skoneczny [9].

Practical experiments however, showed the insufficiency of applying to color images this two-level contrast operator proposed by Angulo, i.e. using the ordering based on Distance to

*e-mail: slaweks@isep.pw.edu.pl 
Reference Color (DRC), as well as three-level contrast operator proposed in [9]. In the first case the output images often looked too sharp, in the second one the quality of the output image was not high enough for some color pictures coming from old movies. Also the details of DRC ordering were not obvious to be fixed (the choice of the reference color is rather arbitrary here), which might lead to different results of morphological operations. Those difficulties suggest the need for more levels in toggle based approach, to increase the contrast in blurred images and to reduce the cartoon-like visual impression of the viewer. In addition to that it is worth emphasizing that, the more experiments on the color images were performed by the author of this paper, using many different ordering schemes the more obvious conclusion appeared that a good, automatic, total ordering method ought to be very useful here.

\section{The multistate toggle contrast sharpener}

3.1. Classical toggle contrast operator. For gray-level images the contrast toggle mapping is defined by using two factors. The first factor is choosing two primitives $\phi_{1}$ and $\phi_{2}$, which are applied to the initial image. The second factor is a proper decision rule, which makes at each point $x=\left(x_{1}, x_{2}\right)$ the output of this mapping toggle between two possibilities $\phi_{1}$ and $\phi_{2}$, depending on which is the closest to the input value of the function $f$ at $x[5,10]$. Angulo [8] extended two-level toggle method for gray-scale image to color image f) with dilation (i.e. $\phi_{1}(\mathbf{f})=\delta_{X}(\mathbf{f})$ ) and erosion (i.e. $\phi_{2}(\mathbf{f})=\epsilon_{X}(\mathbf{f})$ ) as two primitives and he proposed the contrast operator with $X$-ordering applied to the image $\mathbf{f}$ at point $x=\left(x_{1}, x_{2}\right)$ (pixel coordinates in image) as:

$$
\kappa_{X}^{\delta, \epsilon}(\mathbf{f})(x)=\left\{\begin{array}{l}
\delta_{X}(\mathbf{f})(x) \text { if } \\
\left\|\mathbf{f}(x)-\delta_{X}(\mathbf{f})(x)\right\| \leq\left\|\mathbf{f}(x)-\epsilon_{X}(\mathbf{f})(x)\right\| \\
\epsilon_{X}(\mathbf{f})(x) \text { if } \\
\left\|\mathbf{f}(x)-\delta_{X}(\mathbf{f})(x)\right\|>\left\|\mathbf{f}(x)-\epsilon_{X}(\mathbf{f})(x)\right\| .
\end{array}\right.
$$

3.2. Contrast sharpener with odd number of levels. This approach is a generalization of method from [6] (gray-scale images) and Angulo's approach, who proposed a two-state toggle operator for color images. It leads to the toggle contrast operators of any number of levels (called multistate operators). Two-state contrast operation sometimes gives very rough image, so it seems reasonable for each pixel to have the possibility of choosing more levels of colors resulting from the application of different morphological operations.

Suppose we have a color image $\mathrm{f}$. We have also a set of extensive morphological operators $\Phi_{X}^{i}$, and a set of antiextensive morphological operators $\Gamma_{X}^{i}$, where $i=1, \ldots k$. Assume that we use a total ordering of color pixels and let us denote it by $X$. Now we can define the special ratio:

$$
\rho_{2 k, 2 k+1}(\mathbf{f})=\frac{\left\|\sum_{i=1}^{k}\left[\Phi_{X}^{i}(\mathbf{f})-\mathbf{f}\right]\right\|_{2}}{\left\|\sum_{i=1}^{k}\left[\Phi_{X}^{i}(\mathbf{f})-\Gamma_{X}^{i}(\mathbf{f})\right]\right\|_{2}}
$$

We denote the four most often used morphological operations in the following way: $\delta_{X}$-dilation, $\epsilon_{X}$-erosion, $\gamma_{X}$-opening, $\phi_{X}$-closing. Two lower indices are useful because the same formula (2) can be used to build two different contrast enhancing operators. The first one has the odd number of toggle states, the second one the even number of toggle states.

For example (if $k=1$ or $k=2$ ) we may have:

$$
\begin{gathered}
\rho_{2,3}(\mathbf{f})=\frac{\left\|\delta_{X}(\mathbf{f})-\mathbf{f}\right\|_{2}}{\left\|\delta_{X}(\mathbf{f})-\epsilon_{X}(\mathbf{f})\right\|_{2}} \\
\rho_{4,5}(\mathbf{f})=\frac{\left\|\delta_{X}(\mathbf{f})+\phi_{X}(\mathbf{f})-2 \cdot \mathbf{f}\right\|_{2}}{\left\|\delta_{X}(\mathbf{f})+\phi_{X}(\mathbf{f})-\gamma_{X}(\mathbf{f})-\epsilon_{X}(\mathbf{f})\right\|_{2}} .
\end{gathered}
$$

The general equation for $M$-state contrast, where $M$ is an odd number, i.e. $M=2 k+1$ is:

$$
\begin{aligned}
& \begin{cases}\Phi_{X}^{1}(\mathbf{f})(x) & \text { if } \quad 0 \leq \rho(\mathbf{f})(x)<\frac{1}{(2 k+1)} \\
\Phi_{X}^{2}(\mathbf{f})(x) & \text { if } \quad \frac{1}{(2 k+1)} \leq \rho(\mathbf{f})(x)<\frac{2}{(2 k+1)}\end{cases}
\end{aligned}
$$

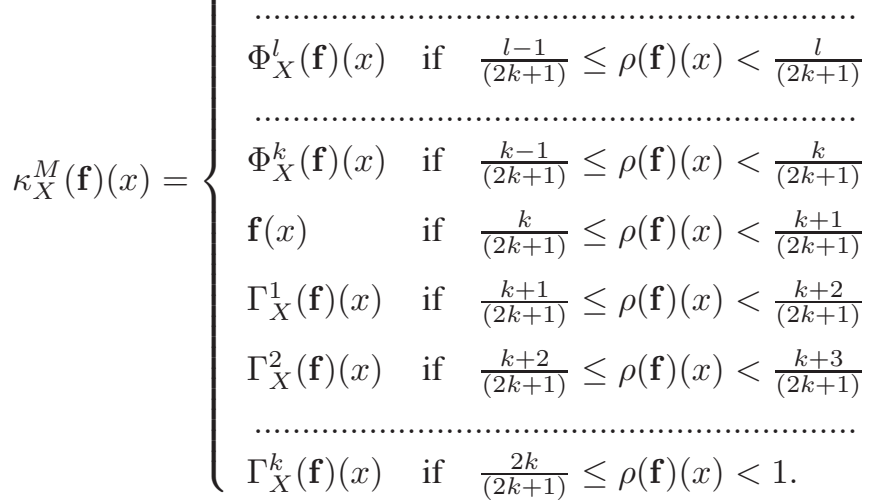

For example for 7-state contrast enhancing operator (i.e. $k=3$ ) we have:

$$
\kappa_{X}^{7}(\mathbf{f})(x)= \begin{cases}\Phi_{X}^{1}(\mathbf{f})(x) & \text { if } \quad 0 \leq \rho(\mathbf{f})(x)<\frac{1}{7} \\ \Phi_{X}^{2}(\mathbf{f})(x) & \text { if } \quad \frac{1}{7} \leq \rho(\mathbf{f})(x)<\frac{2}{7} \\ \Phi_{X}^{3}(\mathbf{f})(x) & \text { if } \quad \frac{2}{7} \leq \rho(\mathbf{f})(x)<\frac{3}{7} \\ \mathbf{f}(x) & \text { if } \quad \frac{3}{7} \leq \rho(\mathbf{f})(x)<\frac{4}{7} \\ \Gamma_{X}^{1}(\mathbf{f})(x) & \text { if } \quad \frac{4}{7} \leq \rho(\mathbf{f})(x)<\frac{5}{7} \\ \Gamma_{X}^{2}(\mathbf{f})(x) & \text { if } \quad \frac{5}{7} \leq \rho(\mathbf{f})(x)<\frac{6}{7} \\ \Gamma_{X}^{3}(\mathbf{f})(x) & \text { if } \quad \frac{6}{7} \leq \rho(\mathbf{f})(x)<1 .\end{cases}
$$

In this particular case the $\Phi_{X}^{i}$ 's and $\Gamma_{X}^{i}$ 's must be chosen in such way that the following relation holds:

$$
\begin{aligned}
& \Gamma_{X}^{3}(\mathbf{f}) \prec \Gamma_{X}^{2}(\mathbf{f}) \prec \Gamma_{X}^{1}(\mathbf{f}) \prec \mathbf{f} \\
& \prec \Phi_{X}^{3}(\mathbf{f}) \prec \Phi_{X}^{2}(\mathbf{f}) \prec \Phi_{X}^{1}(\mathbf{f}),
\end{aligned}
$$

where $\prec-$ means the ordering relation "less or equal" for multivariate data according to the ordering method $X$.

A good choice seems to be the following set of morphological operations: $\Phi_{X}^{1}(\mathbf{f})=\delta_{X}(\mathbf{f})$ - color dilation according to the order $X, \Phi_{X}^{2}(\mathbf{f})=\phi_{X}(\mathbf{f})$ - color closing according to the order $X, \Phi_{X}^{3}(\mathbf{f})=\phi_{X} \gamma_{X} \phi_{X}(\mathbf{f})$ - color close-open-close filter accord. to the order $X, \Gamma_{X}^{1}(\mathbf{f})=\gamma_{X} \phi_{X} \gamma_{X}(\mathbf{f})-$ color 


\section{Morphological sharpening of color images}

open-close-open filter accord. to the order $X, \Gamma_{X}^{2}(\mathbf{f})=\gamma_{X}(\mathbf{f})$ - color opening according to the order $X, \Gamma_{X}^{3}(\mathbf{f})=\epsilon_{X}(\mathbf{f})-$ color erosion according to the order $X$.

\subsection{Choosing increasing and decreasing operators for} multistage toggle contrast. For 3-stage or 5-stage toggle contrast the most natural choice is to take from the set of basic operations: erosion $-\epsilon_{X}$ (anti-extensive), dilation $-\delta_{X}$ (extensive), opening $-\gamma_{X}$ (anti-extensive), closing $-\phi_{X}$ (extensive) and identity operator $I$. For more states than five the problem is more complicated. We know from the morphological theory for gray-level images that the composition of basic morphological ordered filters i.e. opening $-\gamma$ and closing $\phi$ lead to new morphological filters $\gamma \phi, \phi \gamma, \gamma \phi \gamma, \phi \gamma \phi$. For a gray-level case the following ordering relations are always satisfied [11]:

$$
\epsilon \leq \gamma \leq \gamma \phi \gamma \leq \gamma \phi, \quad \phi \gamma \leq \phi \gamma \phi \leq \phi \leq \delta .
$$

Although open-close $\gamma \phi$ and close open $\phi \gamma$ filters give almost the same filtering results they are not equivalent. Moreover, there exists no ordering between $\gamma \phi$ and $\phi \gamma$, nor between $\gamma \phi$ and $I$, nor between $\phi \gamma$ and $I$ [11]. So we cannot use these filters, i.e. $\gamma_{X} \phi_{X}, \phi_{X} \gamma_{X}$, for a color case of multilevel toggle contrast. If we denote by $\prec$ the relation "less or equal" for multivariate data then for the total order $X$ in color images the following relations exist:

$$
\begin{gathered}
\epsilon_{X} \prec \gamma_{X} \prec \gamma_{X} \phi_{X} \gamma_{X} \prec \gamma_{X} \phi_{X}, \\
\phi_{X} \gamma_{X} \prec \phi_{X} \gamma_{X} \phi_{X} \prec \phi_{X} \prec \delta_{X},
\end{gathered}
$$

where $\epsilon_{X}, \delta_{X}, \gamma_{X}, \phi_{X}$ are erosion, dilation, opening and closing respectively, according to the chosen ordering of pixels $X$. So for multistage toggle based contrast of color images we can use, for example, the set $S_{\text {basic1 }}$ consisting of the following morphological operations:

$$
S_{\text {basic } 1}=\left\{\epsilon_{X}, \gamma_{X}, \gamma_{X} \phi_{X} \gamma_{X}, \phi_{X} \gamma_{X} \phi_{X}, \phi_{X}, \delta_{X}\right\} .
$$

We can write down the formula of the general set $S_{G e n}$ built of extensive morphological operators: $\Phi_{X}^{i}$, where $i=$ $1, \ldots m$, and anti-extensive morphological operators $\Gamma_{X}^{j}$, where $j=1, \ldots, n$, as well as the identity operator $I$ as follows:

$$
S_{G e n}=\left\{\Gamma_{X}^{m}, \ldots, \Gamma_{X}^{2}, \Gamma_{X}^{1}, I, \Phi_{X}^{n}, \ldots, \Phi_{X}^{2}, \Phi_{X}^{1}\right\},
$$

where

$$
\Gamma_{X}^{m} \prec \ldots \prec \Gamma_{X}^{2} \prec \Gamma_{X}^{1} \prec I \prec \Phi_{X}^{n} \prec \ldots \prec \Phi_{X}^{2} \prec \Phi_{X}^{1},
$$

which means that $S_{G e n}$ is an ordered set of operators, arranged in ascending order. By using this basic set of ordered operators $S_{\text {basic1 }}$ we can construct some new sets. The elements of all these sets are arranged in ascending order. We can take advantage of these new sets while designing the morphological multilevel toggle based contrast sharpening filters. For instance, if we want to build the contrast of four or five states, we may use the following ascendingly ordered set:

$$
S_{A}^{4,5}=\left\{\epsilon_{X}, \gamma_{X}, I, \phi_{X}, \delta_{X}\right\}
$$

On the other hand, if we prefer to have more-valued contrast sharpening filters, e.g. of six or seven levels we may use another set of operators, for example:

$$
S_{A}^{6,7}=\left\{\epsilon_{X}, \gamma_{X}, \gamma_{X} \phi_{X} \gamma_{X}, I, \phi_{X} \gamma_{X} \phi_{X}, \phi_{X}, \delta_{X}\right\} .
$$

There are many other possibilities of building the sets for operations suited to the multistage toggle contrast. The general rule is that first "half" of the set should consist of antiextensive operators and the second "half" should consist of extensive ones plus identity operator in the middle of the set.

3.4. Contrast sharpener with even number of levels. The general equation for $N$-state contrast, where $N=2 k$, is as follows:

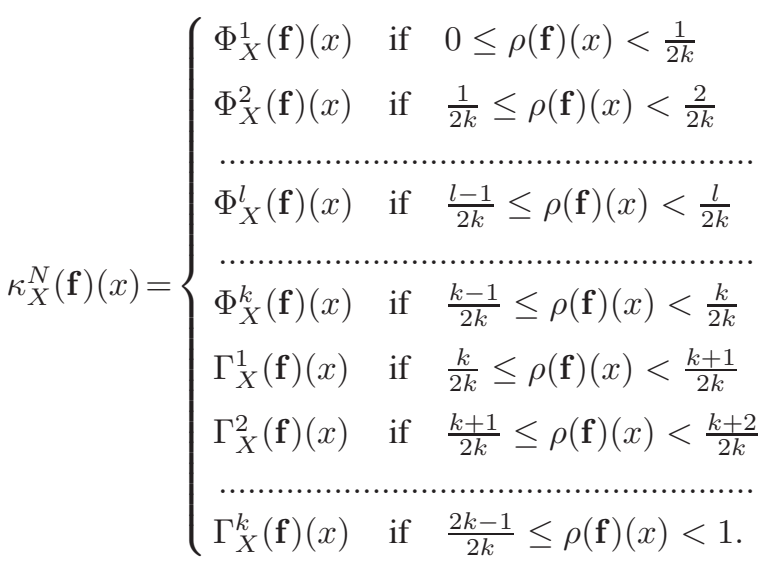

For instance the 6-state contrast operator (i.e. $k=3$ ) can be defined as:

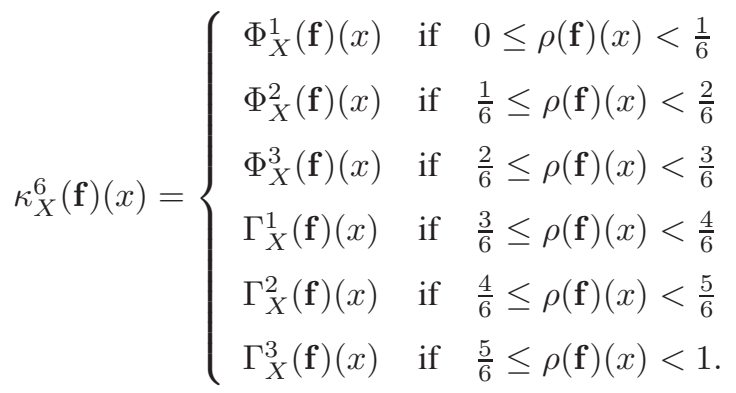

In the latter case we can choose the same set of operations as before, i.e. for odd number of levels contrast operator, or for example the other one like: $\Phi_{X}^{1}(\mathbf{f})=\delta_{X}(\mathbf{f})$ - color dilation according to the order $X, \Phi_{X}^{2}(\mathbf{f})=\phi_{X}(\mathbf{f})$ - color closing according to the order $X, \Phi_{X}^{3}(\mathbf{f})=\phi_{X} \gamma_{X} \phi_{X}(\mathbf{f})$ - color closeopen-close filter according to order $X, \Gamma_{X}^{1}(\mathbf{f})=\gamma_{X} \phi_{X} \gamma_{X}(\mathbf{f})$ - color open-close-open filter according to the order $X$, $\Gamma_{X}^{2}(\mathbf{f})=\gamma_{X}(\mathbf{f})-$ color opening according to the order $X$, $\Gamma_{X}^{3}(\mathbf{f})=\epsilon_{X}(\mathbf{f})-$ color erosion according to the order $X$.

We can see that the basic sets of proper morphological operations are constructed in the same way as in the previous subsection of this paper but without using the identity operator.

\section{Pairwise vector ordering}

4.1. Classical Pairwise vector ordering. The Pairwise Vector Ordering (PVO) proposed in [12] has two stages and is a combination of partial ordering and reduced ordering. The 
first step in this approach requires finding the distance defined as:

$$
\left.d_{0}=\max _{i, j \in\{1,2, \ldots, n\}}\left(\| \mathbf{x}_{i}-\mathbf{x}_{j}\right) \|_{p}\right) .
$$

This pair consists of the maximum and minimum color pixels, although no pixel can unambiguously be identified as either. In the second step these two pixels are removed and once again we search for the maximum distance of two vectors from the set of color pixels, which is, say $d_{1}$. The process is repeated and it results in an ordered set of distances: $d_{0} \geq d_{1} \geq \ldots \geq d_{(n / 2)-1}$. These two stages induce, according to Evans, "full ordering". It is not clear whether he meant total ordering, however it is not difficult to show that the order induced by Pairwise Vector Approach is not a total one but only a pre-ordering! It can be easily demonstrated by the following example. Suppose we use the RGB cube $[0, \ldots, 255] \times[0, \ldots, 255] \times[0, \ldots, 255]$, i.e. the maximal value for each coordinate is 255 . Assume we have the morphological $3 \times 3$ flat structuring element, or a mask. Under this mask we have 9 pixels with the following R,G,B coordinates presented in Table 1. The graphical presentation of these color pixels is shown in Fig. 1.

Table 1

Pixels (R,G,B triplets) from the image under the structuring element

\begin{tabular}{|c|c|c|c|c|c|c|}
\hline $\mathrm{x} 1$ & $\mathrm{x} 2$ & $\mathrm{x} 3$ & & $(255,0,0)$ & $(0,255,0)$ & $(0,0,255)$ \\
\hline $\mathrm{x} 4$ & $\mathrm{x} 5$ & $x 6$ & $=$ & $(0,255,0)$ & $(153,51,17)$ & $(204,51,51)$ \\
\hline $\mathrm{x} 7$ & $\mathrm{x} 8$ & $\mathrm{x} 9$ & & $(85,15,153)$ & $(15,153,102)$ & $(153,85,102)$ \\
\hline
\end{tabular}

a)

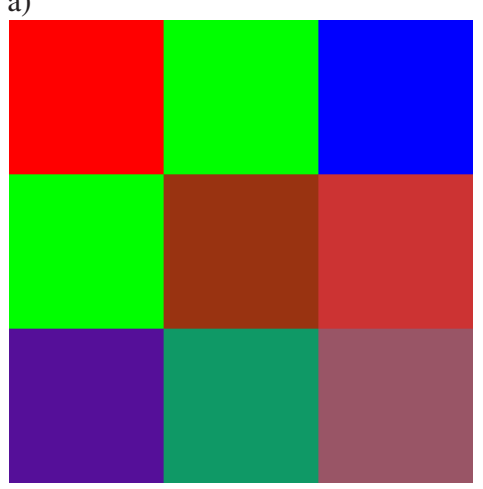

b)
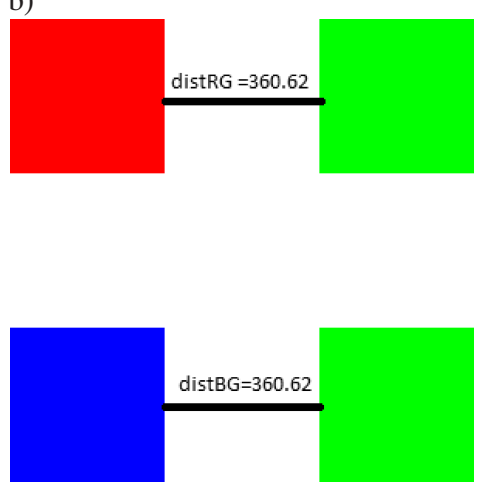

Fig. 1. a) Original 9 pixels, b) two pairs of max. distance - all four pixels have the same distance to the origin of the RGB cube $\mathrm{dR} 0=$ $\mathrm{dG} 0=\mathrm{dB} 0=255$
The first impression might be to solve the situation when the order of two pairs is undecided by using the distance of the pixels in these pairs from the origin of RGB system for ordering the pairs. But, as we can see in this example, all four pixels in these two pairs have the same distance to the origin of the RGB system! We can check it by computing their distances from the origin of the RGB cube: dist(x1,orig) $=\operatorname{distR} 0=255, \operatorname{dist}(\mathrm{x} 2$, orig $)=\operatorname{distG} 0=255, \operatorname{dist}(\mathrm{x} 3$, orig $)=$ $\operatorname{distB} 0=255, \operatorname{dist}(\mathrm{x} 4$, orig $)=\operatorname{distG0}=255$.

All these four pixels are on the same sphere of radius 255 and its center is the origin of the RGB system! Therefore all these four points are equidistant to the origin. So we cannot just use the simple distance of pixels to the origin as the next step in ordering method. We need the additional step in ordering algorithm and this step should be different from computing another distance. The concept proposed by Evans (PVO) simply does not work here! That is why the MPO the Modified Pairwise Ordering is proposed in this paper. Of course it is not the only one possibility. But we have a real total ordering of pixels.

The mutual Euclidean distances of all nine color pixels are given in Table 2. We have two pairs of maximal distances: dis$\mathrm{tRG}=$ distBG $=360.6$. The first pair includes the perfect Red pixel $x 1=[255,0,0]$ and the perfect Green one $x 2=[0,255,0]$. The second pair includes the perfect Blue pixel $x 3=[0,0,255]$ and the perfect Green pixel $x 4=[0,255,0]$. According to the Pairwise Vector Ordering proposed by Evans we should use the pair of maximal distance that includes the minimal and maximal pixel i.e. - erosion and dilation. First of all we have ambiguity here. There are two such pairs of pixels. Which one should we use? In order to overcome this important difficulty the MPO method is proposed. It is presented in details in the next subsection of this paper.

Table 2

The matrix $\mathrm{D}$ of mutual distances of pixels under structuring mask e.g. $\mathrm{D}(\mathrm{x} 1, \mathrm{x} 2)=\sqrt{255^{2}+255^{2}+0^{2}}=255 \sqrt{2}=360.6$

\begin{tabular}{cccccccccc}
\hline \hline & $\mathrm{x} 1$ & $\mathrm{x} 2$ & $\mathrm{x} 3$ & $\mathrm{x} 4$ & $\mathrm{x} 5$ & $\mathrm{x} 6$ & $\mathrm{x} 7$ & $\mathrm{x} 8$ & $\mathrm{x} 9$ \\
\hline $\mathrm{x} 1$ & 0 & 360.6 & 360.6 & 360.6 & 115.3 & 88.3 & 229.2 & 302.3 & 167.4 \\
$\mathrm{x} 2$ & 360.6 & 0 & 360.6 & 0 & 255.6 & 293.0 & 297.0 & 145.0 & 250.4 \\
x3 & 360.6 & 360.6 & 0 & 360.6 & 287.5 & 293.0 & 133.6 & 216.9 & 232.5 \\
x4 & 360.6 & 0 & 360.6 & 0 & 255.6 & 293.0 & 297.0 & 145.0 & 250.4 \\
x5 & 115.3 & 255.6 & 287.5 & 255.6 & 0 & 61.3 & 156.3 & 191.5 & 91.6 \\
x6 & 88.3 & 293.0 & 293.0 & 293.0 & 61.3 & 0 & 160.8 & 220.7 & 79.7 \\
x7 & 229.2 & 297.0 & 134.0 & 297.0 & 156.3 & 160.8 & 0 & 162.9 & 110.1 \\
x8 & 302.3 & 145.0 & 217.0 & 145.0 & 191.5 & 220.7 & 162.9 & 0 & 153.8 \\
x9 & 167.4 & 250.4 & 232.5 & 250.4 & 91.6 & 79.7 & 110.1 & 153.8 & 0 \\
\hline
\end{tabular}

4.2. The Modified Pairwise Ordering. In order to achieve the total ordering utilizing the concept of Pairwise Vector Ordering we propose some modifications. For our purpose we would like to find only the maximum and minimum of this totally ordered set of color pixels. For full 3-D case (i.e. $\left.\mathbf{x}_{\mathbf{i}}=\left(r_{i}, g_{i}, b_{i}\right), \mathbf{x}_{\mathbf{j}}=\left(r_{j}, g_{j}, b_{j}\right)\right)$ the new proposed algorithm (called Modified Pairwise Ordering - (MPO)) is as follows:

1) Find $d_{0}$ defined by Eq. (9). 


\section{Morphological sharpening of color images}

2A) If this is a unique solution (i.e. there is only one pair of maximal distance) then compute the magnitude of both vectors $\mathbf{x}_{\mathbf{m}}, \mathbf{x}_{\mathbf{n}}$.

3a)

$$
\begin{aligned}
& \text { If }\left\|\mathbf{x}_{\mathbf{m}}\right\|_{2} \neq\left\|\mathbf{x}_{\mathbf{n}}\right\|_{2} \\
\epsilon= & \mathbf{x}_{\mathbf{i}},\left\{\mathbf{x}_{\mathbf{i}}:\left\|\mathbf{x}_{\mathbf{i}}\right\|_{2}=\min \left(\left\|\mathbf{x}_{\mathbf{m}}\right\|_{2},\left\|\mathbf{x}_{\mathbf{n}}\right\|_{2}\right)\right\} ; \\
\delta= & \mathbf{x}_{\mathbf{j}},\left\{\mathbf{x}_{\mathbf{j}}:\left\|\mathbf{x}_{\mathbf{j}}\right\|_{2}=\max \left(\left\|\mathbf{x}_{\mathbf{m}}\right\|_{2},\left\|\mathbf{x}_{\mathbf{n}}\right\|_{2}\right)\right\} \\
& \text { end }
\end{aligned}
$$

$$
\begin{aligned}
& \text { If }\left\|\mathbf{x}_{\mathbf{m}}\right\|_{2}=\left\|\mathbf{x}_{\mathbf{n}}\right\|_{2} \\
& \begin{array}{l}
\text { If } \mathbf{x}_{\mathbf{m}}<C L O \\
\mathbf{x}_{\mathbf{n}}(\text { see Eq. (12)) } \\
\epsilon=\mathbf{x}_{\mathbf{m}}, \delta=\mathbf{x}_{\mathbf{n}}
\end{array} \\
& \begin{array}{l}
\text { else } \\
\text { end }
\end{array}
\end{aligned}
$$

$$
\text { END }{ }^{\text {end }}
$$

2B) If there is no unique solution ( which means that two or more distinct pairs within the mask have equal maximal distances between two vectors included in each pair) - let us assume that there are $K$ such pairs. Then the procedure should be as follows:

2B1) Build a new set $A_{K}$ from all the vectors of these $K$ pairs. So now we have:

$$
A_{K}=\left\{\mathbf{x}_{i_{1}}, \mathbf{x}_{j_{1}}, \mathbf{x}_{i_{2}}, \mathbf{x}_{j_{2}}, \ldots, \mathbf{x}_{i_{K}}, \mathbf{x}_{j_{K}}\right\}
$$

2B2) Re-numerate the indices of the vectors in this set according the following rules

$$
\mathbf{x}_{2 l-1}=\mathbf{x}_{i_{l}}, \mathbf{x}_{2 l}=\mathbf{x}_{j_{l}}, \quad l=1, \ldots, K
$$

2B3) Arrange the set $A_{K}$ by applying the Classical Lexicographical Ordering (CLO) method:

$$
\mathbf{x}_{m}<_{C L O} \mathbf{x}_{n} \Longleftrightarrow\left\{\begin{array}{l}
r_{m}<r_{n} \text { or } \\
r_{m}=r_{n} \& g_{m}<g_{n} \text { or } \\
r_{m}=r_{n} \& g_{m}=g_{n} \& b_{m}<b_{n}
\end{array}\right.
$$

where $\{m, n\}=1,2, \ldots, 2 K$

$$
\begin{array}{ll}
\text { If }\left(\mathbf{x}_{\mathbf{m}}<_{C L O} \mathbf{x}_{\mathbf{n}}\right) & \epsilon=\mathbf{x}_{\mathbf{m}}, \delta=\mathbf{x}_{\mathbf{n}} \\
\text { else } & \epsilon=\mathbf{x}_{\mathbf{n}}, \delta=\mathbf{x}_{\mathbf{m}} \\
\text { end } &
\end{array}
$$

END

Suppose that $S_{n}$ is a subset of the RGB color space, which includes $n$ vectors $\mathbf{x}_{1}\left(r_{1}, g_{1}, b_{1}\right), \ldots, \mathbf{x}_{n}\left(r_{n}, g_{n}, b_{n}\right)$, where $n$ is the number of pixels under the morphological flat structuring element.

If $A_{K}$ is still the set of $K$ pairs of pixels of maximal distance - for the case of one such pair only i.e. $K=1$ we have the set $A_{1}-$ then using the MPO method we can define the infimum $(\wedge)$ and supremum $(\vee)$ operators in $S_{n}$ as:

$$
\begin{aligned}
& \mathbf{x}_{\text {low }}\left(r_{\text {low }}, g_{\text {low }}, b_{\text {low }}\right)=\wedge S_{n}=\epsilon=\wedge A_{K}, \\
& \mathbf{x}_{\text {up }}\left(r_{\text {up }}, g_{\text {up }}, b_{\text {up }}\right)=\vee S_{n}=\delta=\vee A_{K} .
\end{aligned}
$$

Properties of the MPO are mostly classical ones. However attention should be paid to the fact that, the opening performed with this ordering method of color pixels is not idempotent nor is closing. Therefore more correctly these types of morphological operations should be called pseudo-opening and pseudo-closing respectively.

\section{Other ordering schemes used for comparison}

There are several types of ordering of multivariate data. One of the first papers presenting ordering taxonomy was written by Barnet [13]. Many different methods of color pixel ordering for using morphological operations have been described [14-17]. In this paper one main ordering scheme the Modified Pairwise Ordering algorithm - is used for obtaining morphological operations. However, for comparison a few other arbitrarily chosen ordering methods are also applied.

\subsection{Classical Lexicographical Ordering (CLO) with the} $V \rightarrow S \rightarrow H$ hierarchy. Lexicographical ordering [18] is especially suitable for arranging pixels (vectors) in the context of color Mathematical Morphology, in combination with image data where a natural or artificial priority order exists among the different bands. The lexicographical ordering preserves the input vectors.

In this paper for HSV color space the model with the hierarchy of importance proposed in [19] is used. We define the following ordering relation as:

$$
\Omega=\left\{\|\cdot\|_{\triangle}^{H S V}, V \uparrow \rightarrow S \uparrow \rightarrow H \uparrow\right\},
$$

where $\uparrow$ means increasing order (e.g. $V \uparrow$ means that $V$-values are ordered from the smallest one up to the greatest one). The relation $\Omega$ is actually the Classical Lexicographical Ordering (CLO).

Two color pixels $\mathbf{x}_{i}$ and $\mathbf{x}_{i}$ can be ordered according the following rule:

$$
\mathbf{x}_{i}<\Omega \mathbf{x}_{j} \Longleftrightarrow\left\{\begin{array}{l}
x_{i}^{V}<x_{j}^{V} \text { or } \\
x_{i}^{V}=x_{j}^{V} \& x_{i}^{S}<x_{j}^{S} \text { or } \\
x_{i}^{V}=x_{j}^{V} \& x_{i}^{S}=x_{j}^{S} \& \\
\quad d\left(x_{i}^{H}, H_{r}\right)<d\left(x_{j}^{H}, H_{r}\right),
\end{array}\right.
$$

where the pixel hue distance to the reference hue $d\left(x_{i}^{H}, H_{r}\right)$ is given by Eq. (15).

\subsection{Hexcone ordering (HO) based on physical meaning of} mixing colors with black and white. Although the Hexcone Ordering (HO) is actually a lexicographic ordering in HVS space using $V, 1-S$ and $H$, this approach is used here due to the interesting idea it is based on - namely the physical meaning of mixing colors with white and black and taking into account the amount of the two last factors [20]. This type of ordering has quite simple physical interpretation: the darker a color the smaller it is considered regardless the hue. The most important component in the HSV color space is the value (luminance) $V$. Now we can define the following ordering relation as:

$$
\Lambda=\left\{\|\cdot\|_{\triangle}^{H S V}, V \uparrow \rightarrow S \downarrow \rightarrow H \uparrow\right\},
$$

where $\triangle$ is the color distance, $\uparrow$ means an increasing order. Two color pixels $\mathbf{x}_{i}$ and $\mathbf{x}_{j}$ can be ordered according the following rule [20]: 
S. Skoneczny

$$
\mathbf{x}_{i}<_{\Lambda} \mathbf{x}_{j} \Longleftrightarrow\left\{\begin{array}{l}
x_{i}^{V}<x_{j}^{V} \text { or } \\
x_{i}^{V}=x_{j}^{V} \& x_{i}^{S}>x_{j}^{S} \text { or } \\
x_{i}^{V}=x_{j}^{V} \& x_{i}^{S}=x_{j}^{S} \& \\
\quad d\left(x_{i}^{H}, H_{r}\right)<d\left(x_{i}^{H}, H_{r}\right),
\end{array}\right.
$$

where pixel hue distance to the reference hue can be expressed as:

$$
d\left(x_{i}^{H}, H_{r e f}\right)=\left\{\begin{array}{l}
\left|x_{i}^{H}-H_{r}\right| \text { if }\left|x_{i}^{H}-H_{r}\right| \leq \pi \\
2 \pi-\left|x_{i}^{H}-H_{r}\right| \text { if }\left|x_{i}^{H}-H_{r}\right|>\pi .
\end{array}\right.
$$

5.3. $\alpha$-trimmed lexicographical extrema. Suppose we are given a vector $\mathbf{x} \in \mathcal{R}^{n}$, that contains the sorted gray level pixels under the filtering window. The $\alpha$-trimmed method suggests us to compute the mean of these pixels by rejecting the $2 \alpha$-extreme points:

$$
\alpha M F(\mathbf{x})=\frac{1}{n-2 \alpha} \sum_{i=\alpha+1}^{n-\alpha} x_{i} .
$$

In the case of multidimensional vectors we can use this approach in a very similar manner by applying it to each dimension in an iterative mode [18]. Having a set $X$ of $k$ vectors and wishing to find the maximum of it, we can start from the first dimension, sorting according to this dimension in an increasing order and then the $[\alpha \times k]$ greatest vectors are kept $(\alpha \in(0,1])$ and considered as the new set $X$. If we repeat this process for each dimension, at each step the initial set of vectors becomes smaller. If more than one vector remain at the end of this procedure, we use the last dimension for obtaining the sought extreme. If we search for a minimum, we can use the same formula but with sorting in a decreasing order. This methodology of finding a "collective" extreme does not constitute an ordering and leads to pseudo-morphological operators.

\subsection{Ordering associated with a distance to the reference} color. Anugulo in [8] proposes color morphological operators for which he defines the reference color, which is analogous to the maximum gray level in gray-scale morphology. Here color dilation tends to move toward this chosen reference color and color erosion tends to move away from it. It can be shown that the reference color must have maximum luminance and maximum saturation to enable ordering of color pixels. We apply this approach to color images in the RGB space. The reference color $\mathbf{x}_{\mathbf{0}}$ is chosen as well as the color distance $\triangle$.

We implemented a version suggested by Angulo in [8], who proposes to complete this primary reduced ordering with a lexicographical cascade. If we define this ordering scheme associated with a reference color and completed with a lexicographical cascade as:

$$
\Gamma=\left\{\|\cdot\|_{\triangle}^{R G B}, \mathbf{x}_{0}=\left(x_{0}^{R}, x_{0}^{G}, x_{0}^{B}\right) ; R \uparrow \rightarrow G \uparrow \rightarrow B \uparrow\right\},
$$

where $\uparrow$ means increasing order, then two color pixels $\mathbf{x}_{i}$ and $\mathbf{x}_{j}$ can be ordered according the following rule:

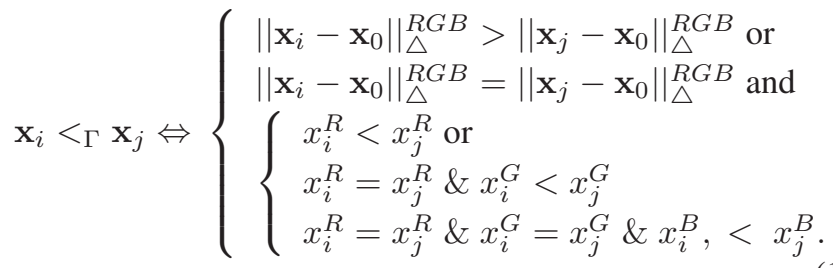

\section{Contrast measure for color image}

There exists some useful local contrast measure in image processing literature. Dhawan et al. [21] define the local contrast for gray level image associated with a pixel at $(i, j)$ and having gray value $x(i, j)$ as:

$$
C(i, j)=\frac{|p(i, j)-a(i, j)|}{|p(i, j)+a(i, j)|}
$$

where

$$
\begin{aligned}
& p(i, j)=\sum_{(i, j) \in N_{1}(i, j)} \frac{x(i, j)}{m^{2}}, \\
& a(i, j)=\sum_{(i, j) \in N_{2}(i, j)} \frac{x(i, j)}{8 m^{2}},
\end{aligned}
$$

where $N_{1}(i, j)$ is an $m \times m$ neighborhood of $(i, j)$ and $N_{2}(i, j)$ is $3 m \times 3 m$ neighborhood of $(i, j)$ which excludes $N_{1}(i, j)$.

For a color image in RGB space we can define:

$$
C^{R G B}(i, j)=\sqrt{\left.\left.\left[C^{R}(i, j)\right]^{2}+C^{G}(i, j)\right]^{2}+C^{B}(i, j)\right]^{2}},
$$

where

$$
\begin{array}{ll}
C^{l}(i, j)=\frac{\left|p^{l}(i, j)-a^{l}(i, j)\right|}{\left|p^{l}(i, j)+a^{l}(i, j)\right|} ; & l=\{R, G, B\}, \\
p^{l}(i, j)=\sum_{(i, j) \in N_{1}(i, j)} \frac{x^{l}(i, j)}{m^{2}} ; & l=\{R, G, B\},
\end{array}
$$

and

$$
a^{l}(i, j)=\sum_{(i, j) \in N_{2}(i, j)} \frac{x^{l}(i, j)}{8 m^{2}} ; \quad l=\{R, G, B\} .
$$

Finally, we can write the mean contrast measure for the whole color image in the RGB coordinates as:

$$
M C M=\frac{1}{K L}\left(\sum_{i=1}^{K} \sum_{j=1}^{L} C^{R G B}(i, j)\right) .
$$

where $K, L$ are dimensions of the processed image.

\section{Experiments}

Experiments were performed with 15 different color images. They are presented in Fig. 2. Among them there were: blurred images taken from old movies, other images of rather bad contrast: e.g. church painting image with low contrast, as well as images that were blurred artificially from original pictures of relatively good visual quality. Eight different contrast sharpening operators based on toggle mappings, including multilevel 
Morphological sharpening of color images

toggles of different numbers of levels varying from 2 to 7 , were used. They were: K2DE, K2CO, K3DIE, K3CIO, K4, $\mathrm{K} 5, \mathrm{~K} 6, \mathrm{~K} 7$. Each of them is described in details further in this section in terms of morphological operations it uses.

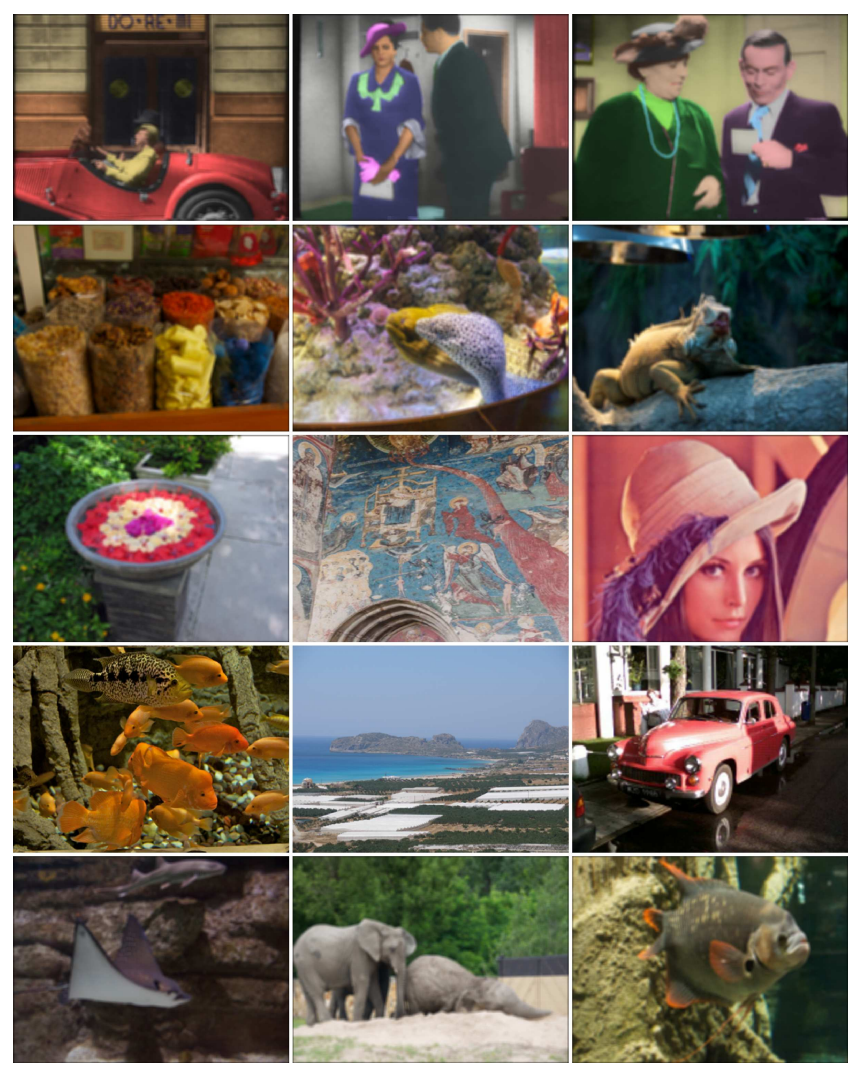

Fig. 2. The whole set of testing blurred images of low contrast

Five different ordering methods were used to arrange color pixels. The first one was the Modified Pairwise Ordering (MPO) in the RGB space. All the contrasted images presented in this section of the paper were obtained by using this method. The second method was the approach based on the Distance to the Reference Color (DRC). Next two different ordering methods in HSV were applied: the Classical Lexicographic Ordering (CLO) with hierarchy of importance: V,S,H, and the Hexcone Ordering (HO) with V,1-S,H hierarchy. Finally the $\alpha$-trimmed method was used. A flat color morphological structuring element of $5 \times 5$ pixels was used.

Most of the proposed contrast enhancing operators increase the mean contrast measure used in this paper taken from the literature [21,22]. Although the mean of all local contrast measures calculated for the whole image is not a uniquely decisive parameter for estimating the overall contrast of the image, it should be seriously taken into account in the process of estimating the quality of contrasted images. In most cases the increase of the contrast measure taken from literature for all images subjected to the two-state operators (erosion and dilation) $\triangle K 2 D E-$ an increase of the average contrast measure - is the highest one for all five types of orderings (MPO, DRC, CLO, $\alpha$-trimmed, HO). The set of all images sharpened by KON2DE is presented in Fig. 3. The advantage of KON2DE sharpening is illustrated graphically by the highest bars in Fig. 4. However, the images obtained by application of K2DE, i.e. two-state contrast with dilation and erosion as basic operations, sometimes look a little bit unnatural, like cartoon images rather than realistic ones. We can see it in Fig. 5 - it concerns in particular the woman driving a car.

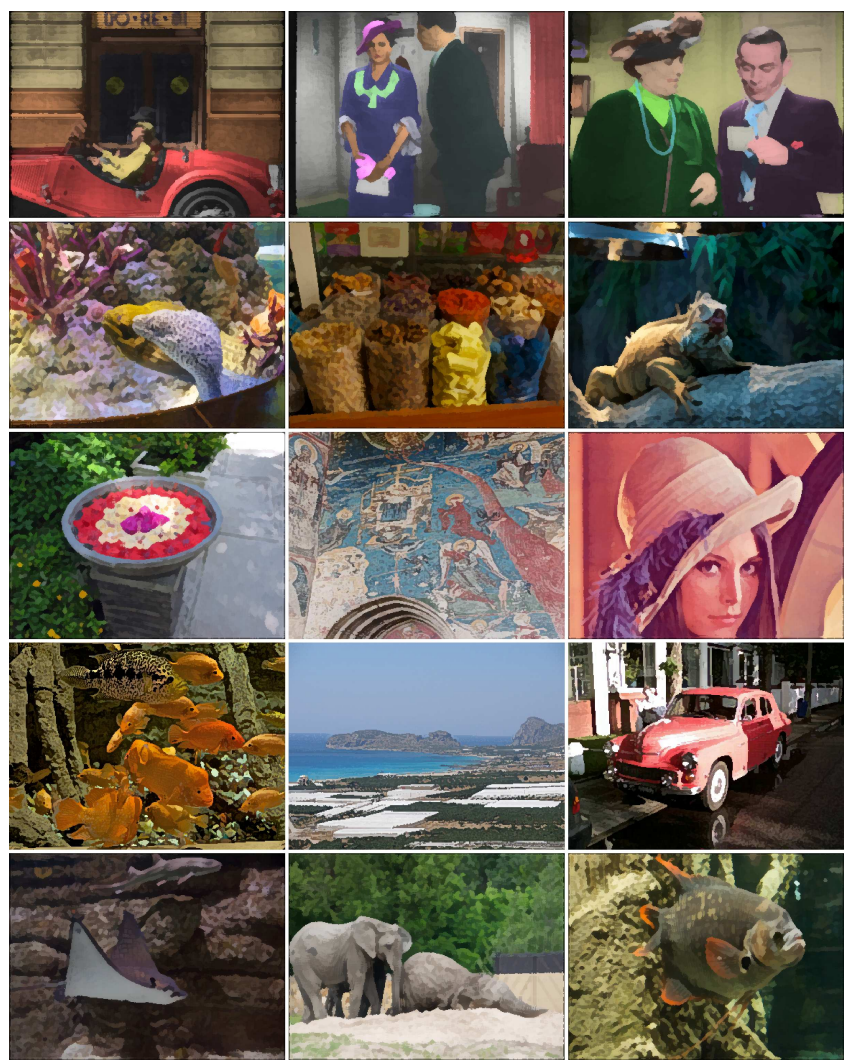

Fig. 3. The whole set of images after sharpening by K2DE operator

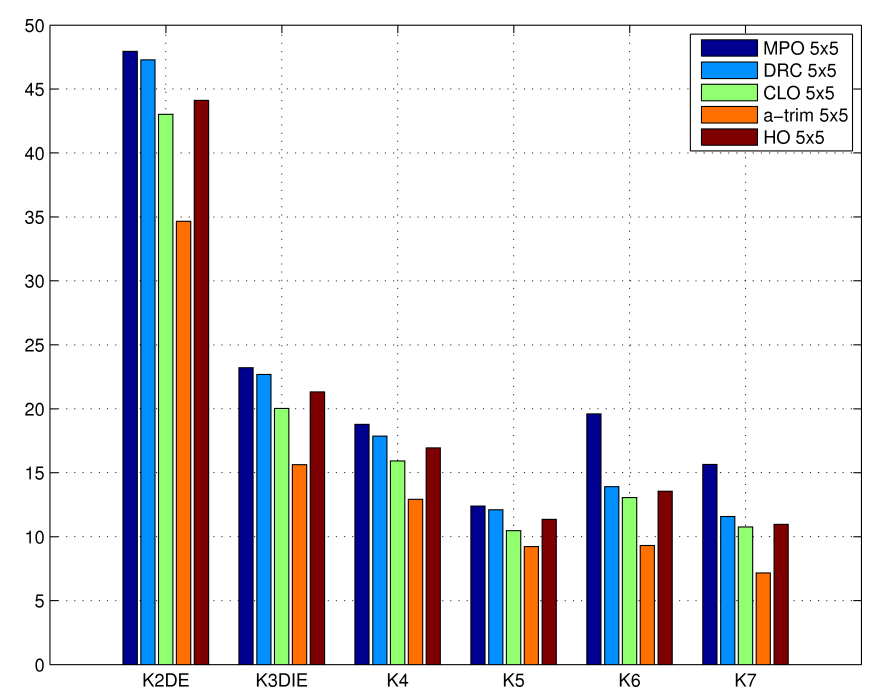

Fig. 4. Bars showing the percentage increase of averaged contrast measure $M C M$ 

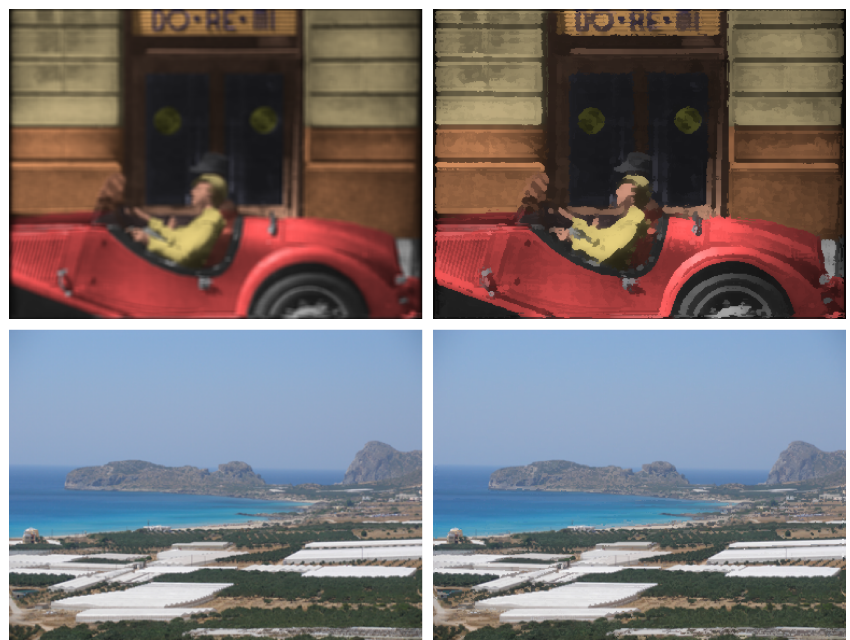

Fig. 5. Images sharpened: by K2DE (right image in the upper row) and by $\mathrm{K} 2 \mathrm{CO}$ (right image in the lower row). Blurred images are in the left column

On the other hand, another two-state contrast operator $\mathrm{K} 2 \mathrm{OC}$ based on two primitives - opening and closing for the flat structuring element increases the contrast measure insufficiently.

Both the original image and the image sharpened by $\mathrm{K} 2 \mathrm{CO}$ are practically visually the same and we can see it in Fig. 5. We need at least $10 \%$ of contrast increase to use $\mathrm{K} 2 \mathrm{CO}$ effectively. The results of sharpening images by twostate contrast operators, i.e. by KON2DE and $\mathrm{KON} 2 \mathrm{CO}$, are presented in Fig. 5. In Table 3 we can see the $M C M$ coefficients for these operators. As concerns the three state operator for contrast enhancement with three chosen operations: erosion, dilation and identity transformation, K3DIE gives the promising results. The average increase of contrast coefficients here was high enough - over $20 \%$.

Table 3

$M C M$ increase for sharpening by $\mathrm{K} 2 \mathrm{DE}$ and $\mathrm{K} 2 \mathrm{CO}$

\begin{tabular}{lccc}
\hline \hline $\begin{array}{c}\text { Order } \\
\text { of pixels }\end{array}$ & $\begin{array}{c}\text { Low } \\
\text { MCM }\end{array}$ & $\begin{array}{c}\text { High } \\
\text { MCM }\end{array}$ & $\begin{array}{c}\text { Average } \\
\text { MCM }\end{array}$ \\
\hline$M P O$ & K2DE & \\
\hline$D R C$ & 29.14 & 62.93 & 47.95 \\
\hline$C L O$ & 27.18 & 62.77 & 47.29 \\
\hline$\alpha-$ trim & 23.13 & 58.65 & 43.02 \\
\hline$H O$ & 18.24 & 46.84 & 34.65 \\
\hline \multicolumn{5}{c}{ K2CO } \\
\hline$M P O$ & 23.76 & 61.61 & 44.11 \\
\hline$D R C$ & 1.28 & 10.34 & 4.73 \\
\hline$C L O$ & -0.13 & 4.90 & 1.21 \\
\hline$\alpha-$ trim & -0.16 & 3.92 & 0.71 \\
\hline$H O$ & -6.94 & -1.81 & -4.18 \\
\hline
\end{tabular}

The contours of all objects are satisfactory sharp - the blur is practically invisible. The image sharpened by K3DIE looks more natural and it is visually acceptable. It is also quite obvious that for an image sharpened by K3DIE the mean contrast measure is generally smaller than the measure for the same image sharpened by K2DE. The reason is that in the first case we sometimes used original blurred pixels by using identity operations. For images sharpened by K3CIO (closing, opening and identity operators) we can see that the sharpening improvements are not large enough to be applied in practice. Again the increases of MCM for all 5 types of pixel orderings are smaller than $10 \%$. This is definitely too less to use this operator in practice for image processing purposes. As we can clearly observe here opening and closing were not successful in two- nor three-level contrast operator for all five types of applied ordering. We can see the visual results of applying two three state operators in Fig. 6. All the numerical results showing the $M C M$ coefficient increases for both three state contrast sharpening operators KON3DIE and KON3CIO are presented in Table 4.
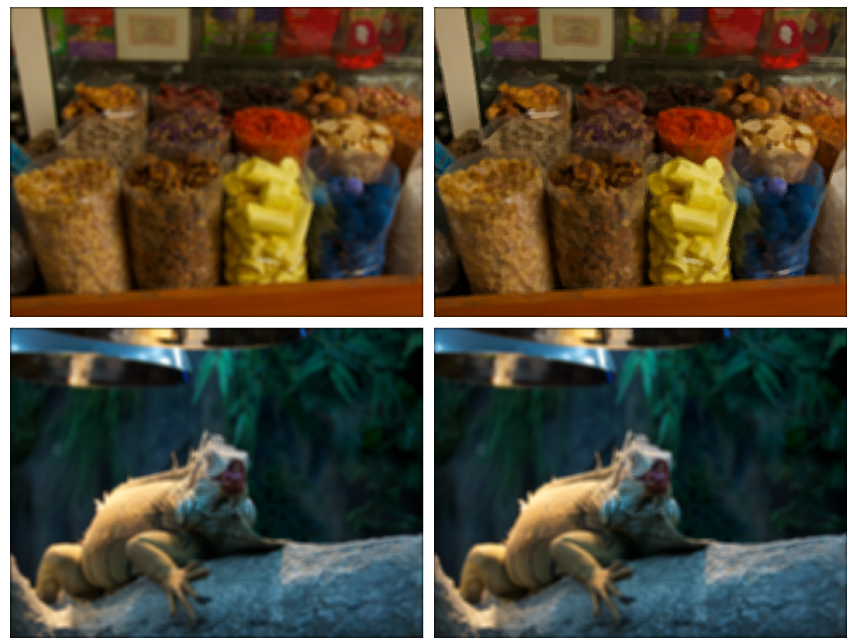

Fig. 6. Images sharpened: by K3DIE (right image in the upper row) and by $\mathrm{K} 3 \mathrm{CIO}$ (right image in the lower row). Blurred images are in the left column

Table 4

$M C M$ increase for sharpening by $\mathrm{K} 3 \mathrm{DIE}$ and $\mathrm{K} 3 \mathrm{CIO}$

\begin{tabular}{lccc}
\hline $\begin{array}{c}\text { Order } \\
\text { of pixels }\end{array}$ & $\begin{array}{c}\text { Low } \\
\text { MCM }\end{array}$ & $\begin{array}{c}\text { High } \\
\text { MCM }\end{array}$ & $\begin{array}{c}\text { Average } \\
\text { MCM }\end{array}$ \\
\hline$M P O$ & \multicolumn{2}{c}{ K3DIE } \\
\hline$D R C$ & 14.70 & 27.84 & 23.21 \\
\hline$C L O$ & 12.57 & 27.68 & 22.68 \\
\hline$\alpha-$ trim & 13.74 & 25.54 & 20.02 \\
\hline$H O$ & 9.10 & 20.03 & 15.63 \\
\hline \multicolumn{5}{c}{ K3CIO } & 21.34 \\
\hline$M P O$ & 11.52 & 8.07 & 3.81 \\
\hline$D R C$ & 1.07 & 0.80 & 3.33 \\
\hline$C L O$ & -0.33 & 0.28 & 2.55 \\
\hline$\alpha-$ trim & -0.13 & -1.30 & -3.05 \\
\hline$H O$ & -5.54 & 3.85 & 0.77 \\
\hline
\end{tabular}

In designing the four-state operator K4 for contrast enhancement the following operations are used: dilation, erosion, closing and opening. The results for K4 operator, in terms of percentage contrast increase, are quite impressive, and again the leader is MPO based sharpener (Avg. MPO $18.78 \%$ vs. $D R C-17.87 \%$. The image quality is quite high, with clear details presented in Fig. 7. 
Morphological sharpening of color images
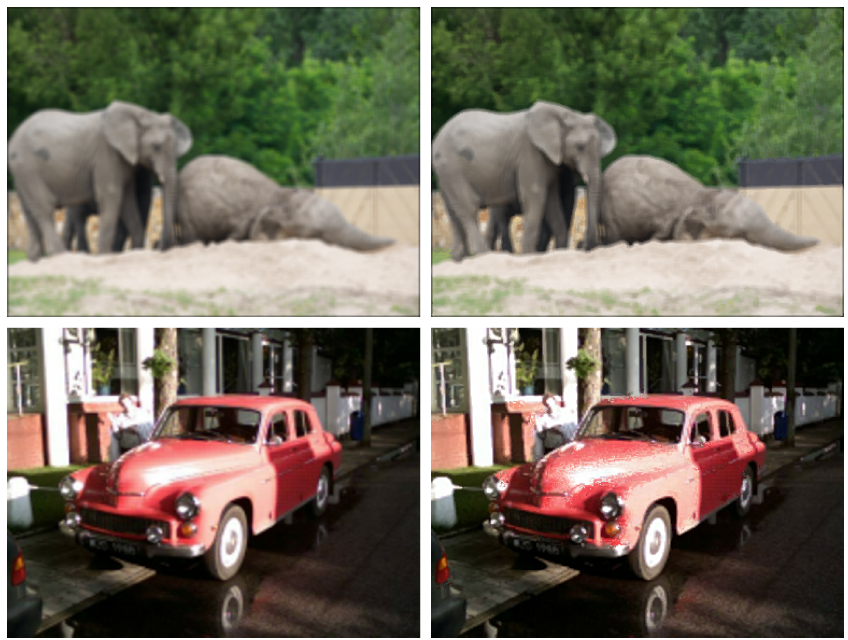

Fig. 7. Images sharpened: by K4 (right image in the upper row) and by $\mathrm{K} 5$ (right image in the lower row). Blurred images are in the left column

We can say that this four state contrast sharpening operator seems to be good enough to be applied to full spectrum of color images of weak contrast. For five - state contrast sharpener $\mathrm{K} 5$ in addition to the same set of transformations like for K4 (i.e. dilation, erosion, closing and opening) the identity operation (I) is utilized. The numerical coefficients for $\mathrm{K} 4$ and $\mathrm{K} 5(M C M)$ are presented in tables shown in Table 5. In this case the improvement in visual quality of the blurred image after applying K5 operator was acceptable. The visual impression of contrast improvement by $\mathrm{K} 5$ is quite clear (see Fig. 7).

Table 5

$M C M$ increase for sharpening by $\mathrm{K} 4$ and $\mathrm{K} 5$

\begin{tabular}{|c|c|c|c|}
\hline $\begin{array}{c}\text { Order } \\
\text { of pixels }\end{array}$ & $\begin{array}{r}\text { Low } \\
\text { MCM }\end{array}$ & $\begin{array}{l}\text { High } \\
\text { MCM }\end{array}$ & $\begin{array}{c}\text { Average } \\
\text { MCM }\end{array}$ \\
\hline \multicolumn{4}{|c|}{ K4 } \\
\hline$M P O$ & 12.89 & 26.49 & 18.78 \\
\hline$D R C$ & 8.25 & 22.66 & 17.87 \\
\hline$C L O$ & 7.26 & 19.91 & 15.93 \\
\hline$\alpha-$ trim & 4.12 & 16.72 & 12.92 \\
\hline $\mathrm{HO}$ & 7.85 & 19.77 & 16.95 \\
\hline \multicolumn{4}{|c|}{ K5 } \\
\hline$M P O$ & 7.85 & 16.67 & 12.39 \\
\hline$D R C$ & 5.04 & 16.86 & 12.11 \\
\hline$C L O$ & 4.19 & 13.51 & 10.48 \\
\hline$\alpha-$ trim & 5.19 & 12.60 & 9.22 \\
\hline $\mathrm{HO}$ & 4.78 & 14.71 & 11.36 \\
\hline
\end{tabular}

In the case of six-level contrast operator K6 each pixel can achieve one of the values which are the result of one of the six morphological operations: erosion, opening, opening followed by closing then opening again, closing, closing followed by opening then closing again and dilation.

For contrast enhancement by the seven - state contrast sharpener $\mathrm{K} 7$ each pixel in the output sharpened image can achieve one of the values which are the result of the identity transformation performed on the input image, which is the pixel of the original image or the result of one of the six morphological operations: erosion, opening, opening followed by closing then opening again, closing, closing followed by opening then closing again and dilation. Both sharpening operators: K6 - applied to the image of an old church painting and $\mathrm{K} 7$ - applied to blurred fish image give the results presented in Fig. 8. Details of the contrasted image are sharper than those of the original one. All the numerical results of MCM coefficient increases for $\mathrm{K} 6$ and $\mathrm{K} 7$ can be seen in Table 6.
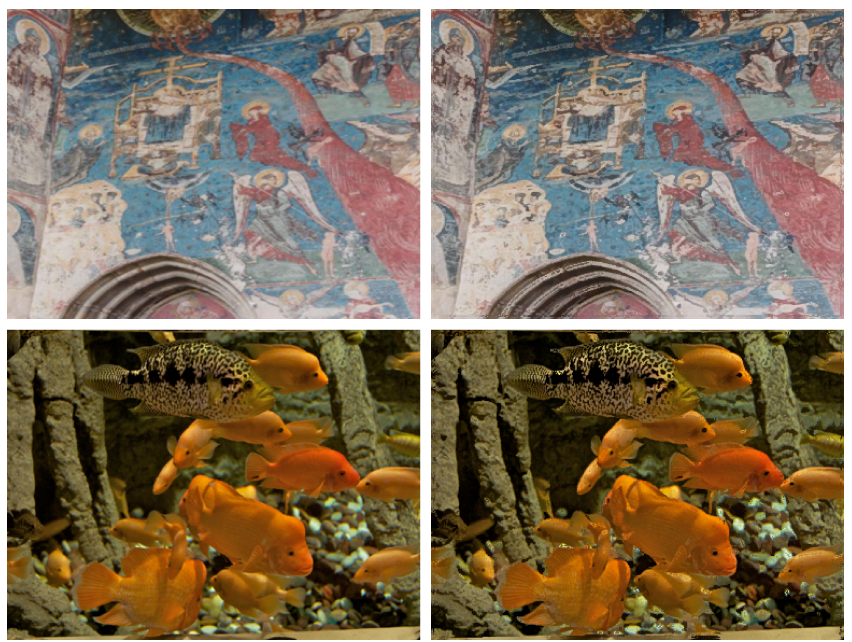

Fig. 8. Images sharpened: by K6 (right image in the upper row) and by $\mathrm{K} 7$ (right image in the lower row). Blurred images are in the left column

Table 6

$M C M$ increase for sharpening by $\mathrm{K} 6$ and $\mathrm{K} 7$

\begin{tabular}{|c|c|c|c|}
\hline $\begin{array}{c}\text { Order } \\
\text { of pixels }\end{array}$ & $\begin{array}{c}\text { Low } \\
\text { MCM }\end{array}$ & $\begin{array}{l}\text { High } \\
\text { MCM }\end{array}$ & $\begin{array}{c}\text { Average } \\
\text { MCM }\end{array}$ \\
\hline \multicolumn{4}{|c|}{ K6 } \\
\hline$M P O$ & 11.66 & 32.29 & 19.59 \\
\hline$D R C$ & 7.53 & 18.12 & 13.91 \\
\hline$C L O$ & 6.81 & 16.84 & 13.06 \\
\hline$\alpha-$ trim & 1.13 & 16.29 & 9.31 \\
\hline $\mathrm{HO}$ & 7.26 & 17.42 & 13.56 \\
\hline \multicolumn{4}{|c|}{ K7 } \\
\hline$M P O$ & 9.33 & 24.68 & 15.64 \\
\hline$D R C$ & 5.82 & 15.53 & 11.58 \\
\hline$C L O$ & 5.24 & 13.51 & 10.76 \\
\hline$\alpha-$ trim & -1.83 & 11.45 & 7.17 \\
\hline $\mathrm{HO}$ & 5.56 & 14.69 & 10.96 \\
\hline
\end{tabular}

In the cases of six-state and seven-state contrast operators the numerical results were surprisingly good. The domination of MPO - based K6 and K7 image sharpening operators over operators based on other ordering methods is exceptionally clear in this case. For example let us consider here the following results:

K6: (Avg. $M P O-19.59 \%$ vs. $D R C-13.91 \%)$;

K7: (Avg. $M P O-15.64 \%$ vs. $D R C-11.58 \%$ ).

The visual quality of both images is quite sufficiently improved from the point of view of human viewer. 


\section{Conclusions}

In this paper the multilevel toggle based contrast sharpener operator has been proposed to enhance the contrast of multivalued images. The effects of its use have been illustrated by many examples of sharpened color images as well as in many tables of contrast coefficients. As concerns the generalized (i.e. multilevel) toggle contrast for colors it offers a wide spectrum of image sharpening possibilities. We have quite large flexibility both in choosing the number of levels as well in building the basic sets of morphological operators that can be used here. It is possible to sharpen many different images to a variable extent, according to need.

The contrast increasing ability, in the sense of their contrast measures of the sharpeners of odd - number of levels are a little bit smaller than of those with even - number of levels. As it was already mentioned there is at least one simple explanation of this phenomenon. These are blurred original pixels - being the result of the identity operator application to the original blurred image. We can also observe the superiority of MPO based sharpeners to their counterparts based on DRC, CLO, HO and $\alpha$-trimmed methods. It is small for $\mathrm{K} 2 \mathrm{DE}(0.7 \%$ more than for DRC), medium for $\mathrm{K} 2 \mathrm{CO}(3 \%$ more than for DRC), small for K3DIE (about $0.6 \%$ more than for DRC), small for: K3CIO $(0.5 \%)$, K4 (0.9\%), K5 (0.3\%), high for K6 $(+5.5 \%)$ and $\mathrm{K} 7(4 \%)$.

As the final remark we can say that the MPO algorithm was the most successful approach as concerns the numerical increase of contrast measures comparing with DRC, CLO, $\mathrm{HO}$ and $\alpha$-trimmed.

The final recommendation for an image processing engineer who wants to use the multistage toggle contrast in order to enhance images he processes can be expressed as follows. As concerns the pixel ordering problem, four methods are recommended namely: MPO, DRC, CLO and HO. The first two approaches are slightly better than the two last methods. But still CLO and HO lead to acceptable results. So these four methods of pixel ordering can be applied to practical problems. The alpha-trimmed ordering is the worst one (significantly worse than others) and should be rejected. The K2DE operator gives the highest increase of the $M C M$ (over $40 \%$ ), but it is responsibility of the image processing engineer to judge its visual quality, sometimes a little bit unnatural. The author of this paper prefers K3DIE, K4, and K6. For them practically for all tested images results are quite satisfactory. $\mathrm{K} 5$ and $\mathrm{K} 7$ are often acceptable but sometimes the contrast is still visually too low.

\section{Proof that the MPO is a total order}

We show that the MPO is a total ordering of color pixels. But before that we need to explain some notations that are used further.

$d_{1, k}=d\left(\mathbf{x}_{1}, \mathbf{x}_{k}\right)$ - is the greatest possible distance of pixel $\mathbf{x}_{1}$ from all the pixels in the set $A$ (in this case this distance is to pixel $\mathbf{x}_{k} \in A$ ). $d_{2, l}=d\left(\mathbf{x}_{2}, \mathbf{x}_{l}\right)-$ is the greatest possible distance of pixel $\mathrm{x}_{2}$ from all the pixels in the set $A$ (in this case this distance is to pixel $\mathbf{x}_{l} \in A$ ).

$d_{3, m}=d\left(\mathbf{x}_{3}, \mathbf{x}_{m}\right)$ - is the greatest possible distance of pixel $\mathbf{x}_{3}$ from all the pixels in the set $A$ (in this case this distance is to pixel $\mathbf{x}_{m} \in A$ ).

1) First we check reflexivity:

$$
\begin{gathered}
\mathbf{x}_{1} \leq_{M P O} \mathbf{x}_{1} \Leftrightarrow\left(d_{1, k}<d_{1, k}\right) \vee\left[\left(d_{1, k}=d_{1, k}\right)\right. \\
\left.\wedge\left(\mathbf{x}_{1} \leq_{l e x} \mathbf{x}_{\mathbf{1}}\right)\right] \Leftrightarrow \mathbf{x}_{1}={ }_{\text {lex }} \mathbf{x}_{1},
\end{gathered}
$$

which is true. Therefore, the MPO relation is reflexive.

2) Next we check if this ordering relation is antisymmetric:

$$
\begin{aligned}
\mathbf{x}_{1} \leq_{M P O} \mathbf{x}_{2} \Leftrightarrow & \left(d_{1, k}<d_{2, l}\right) \vee\left[\left(d_{1, k}=d_{2, l}\right)\right. \\
& \left.\wedge\left(\mathbf{x}_{1} \leq_{l e x} \mathbf{x}_{2}\right)\right], \\
\mathbf{x}_{2} \leq_{M P O} \mathbf{x}_{1} \Leftrightarrow & \left(d_{2, l}<d_{1, k}\right) \vee\left[\left(d_{2, l}=d_{1, k}\right)\right. \\
& \left.\wedge\left(\mathbf{x}_{2} \leq_{l e x} \mathbf{x}_{1}\right)\right] .
\end{aligned}
$$

So if

$$
\begin{gathered}
\left\{\mathbf{x}_{1} \leq_{M P O} \mathbf{x}_{2}\right\} \wedge\left\{\mathbf{x}_{2} \leq_{M P O} \mathbf{x}_{1}\right\} \Leftrightarrow \\
\left\{\left(d_{1, k}<d_{2, l}\right) \vee\left[\left(d_{1, k}=d_{2, l}\right) \wedge\left(\mathbf{x}_{1} \leq_{l e x} \mathbf{x}_{2}\right)\right]\right\} \wedge \\
\left\{\left(d_{2, l}<d_{1, k}\right) \vee\left[\left(d_{2, l}=d_{1, k}\right) \wedge\left(\mathbf{x}_{2} \leq_{l e x} \mathbf{x}_{1}\right)\right]\right\} \Leftrightarrow \\
\left(d_{1, k}<d_{2, l}\right) \wedge\left\{( d _ { 2 , l } < d _ { 1 , k } ) \vee \left[\left(d_{2, l}=d_{1, k}\right) \wedge\right.\right. \\
\left.\left.\left(\mathbf{x}_{2} \leq_{l e x} \mathbf{x}_{1}\right)\right]\right\} \vee\left[\left(d_{1, k}=d_{2, l}\right) \wedge\left(\mathbf{x}_{1} \leq_{l e x} \mathbf{x}_{2}\right)\right] \wedge \\
\left\{\left(d_{2, l}<d_{1, k}\right) \vee\left[\left(d_{2, l}=d_{1, k}\right) \wedge\left(\mathbf{x}_{2} \leq_{l e x} \mathbf{x}_{1}\right)\right]\right\} \Leftrightarrow \\
\left\{[ ( d _ { 1 , k } < d _ { 2 , l } ) \wedge ( d _ { 2 , l } < d _ { 1 , k } ) ] \vee \left[\left(d_{1, k}<d_{2, l}\right) \wedge\right.\right. \\
\left.\left.\left(d_{1, k}=d_{2, l}\right) \wedge\left(\mathbf{x}_{1} \leq_{l e x} \mathbf{x}_{2}\right)\right]\right\} \\
\vee\left\{\left[\left(d_{1, k}=d_{2, l}\right) \wedge\left(x_{1} \leq_{l e x} x_{2}\right) \wedge\left(d_{2, l}<d_{1, k}\right)\right] \vee\right. \\
{\left[\left(d_{1, k}=d_{2, l}\right) \wedge\left(\mathbf{x}_{1} \leq_{l e x} \mathbf{x}_{2}\right) \wedge\right.} \\
\left.\left.\left(d_{2, l}=d_{1, k}\right) \wedge\left(\mathbf{x}_{2} \leq_{l e x} \mathbf{x}_{1}\right)\right]\right\} \\
=0 \vee 0 \vee 0 \vee\left[( d _ { 1 , k } = d _ { 2 , l } ) \wedge ( \mathbf { x } _ { 1 } \leq _ { l e x } \mathbf { x } _ { 2 } ) \wedge \left(\mathbf{x}_{2} \leq l e x\right.\right. \\
=\left[( d _ { 1 , k } = d _ { 2 , l } ) \wedge \left(\mathbf{x}_{1}=l e x\right.\right. \\
\left.\left.\mathbf{x}_{2}\right)\right] \Leftrightarrow \mathbf{x}_{1}={ }_{M P O} \mathbf{x}_{2} .
\end{gathered}
$$

Therefore the MPO relation is antisymmetric.

3) Finally, we show the property of transitivity:

$$
\begin{gathered}
\left\{\left(\mathbf{x}_{1} \leq_{M P O} \mathbf{x}_{2}\right)\right\} \wedge\left\{\mathbf{x}_{2} \leq_{M P O} \mathbf{x}_{3}\right\} \Leftrightarrow \\
\left\{\left(d_{1, k}<d_{2, l}\right) \vee\left[\left(d_{1, k}=d_{2, l}\right) \wedge\left(\mathbf{x}_{1} \leq_{l e x} \mathbf{x}_{2}\right)\right]\right\} \wedge \\
\left\{\left(d_{2, l}<d_{3, m}\right) \vee\left[\left(d_{2, l}=d_{3, m}\right) \wedge\left(\mathbf{x}_{2} \leq_{l e x} \mathbf{x}_{3}\right)\right]\right\} \Leftrightarrow \\
\left\{\left(d_{1, k}<d_{2, l}\right) \wedge\left(d_{2, l}<d_{3, m}\right)\right\} \\
\left.\left.\left(\mathbf{x}_{1} \leq_{l e x} \mathbf{x}_{2}\right)\right] \wedge\left(d_{2, l}<d_{3, m}\right)\right\} \vee\left\{\left(d_{1, k}=d_{2, l}\right) \wedge\right. \\
\left.\left[\left(d_{2, l}=d_{3, m}\right) \wedge\left(\mathbf{x}_{2, l} \leq_{l e x} \mathbf{x}_{3}\right)\right]\right\} \\
\left.\left(\mathbf{x}_{1} \leq_{l e x} \mathbf{x}_{2}\right)\right] \wedge\left[\left(d_{2, l}=d_{3, m}\right) \wedge\left(d_{1, k}=d_{2, l}\right) \wedge\right. \\
\left.\left.\left(d_{1, k} \leq_{l e x} \mathbf{x}_{3}\right)\right]\right\} \Leftrightarrow \\
\left.\left(\mathbf{x}_{1, m} \leq_{l e x} \mathbf{x}_{2}\right)\right] \vee\left[\left(d_{1, k}<d_{3, m}\right) \wedge\right. \\
\left.\left(\mathbf{x}_{2} \leq_{l e x} \mathbf{x}_{3}\right)\right] \vee\left[\left(d_{1, k}=d_{3, m}\right) \wedge\right. \\
\left.=(\text { By redundancy law }) \wedge\left(\mathbf{x}_{1} \leq_{l e x} \mathbf{x}_{3}\right)\right] \\
{\left[\left(d_{1, k}=d_{3, m}\right) \wedge\left(\mathbf{x}_{1} \leq_{l e x} \mathbf{x}_{3}\right)\right] \Longleftrightarrow} \\
\mathbf{x}_{1} \leq_{M P O} \mathbf{x}_{3} .
\end{gathered}
$$

Therefore the MPO relation is transitive.

So we can say that the MPO is a total order. 
Morphological sharpening of color images

\section{REFERENCES}

[1] F. Stanco, S. Battiato, and G. Gallo, Digital Imaging for Cultural Heritage Preservation: Analysis, Restoration, and Reconstruction of Ancient Artworks, CRC Press, London, 2011.

[2] T. Markiewicz, M. Dziekiewicz, S. Osowski, M. Maruszynski, W. Kozlowski, and R. Boguslawska-Walecka, "Thresholding techniques for segmentation of atherosclerotic plaque and lumen areas in vascular arteries" Bull. Pol. Ac.: Tech. 63 (1), 269-280 (2015).

[3] S.A. Mahmoudi, M. Kierzynka, P. Manneback, and K. Kurowski, "Real-time motion tracking using optical flow on multiple GPUs", Bull. Pol. Ac.: Tech. 62 (1), 139-150 (2014).

[4] H. Kramer and J. Bruckner, "Iteration of non-linear transformation for enhancement of digital image", Pattern Recogn. 7, 53-58 (1975).

[5] J. Serra, "Toggle mappings", in From Pixels to Features, J.C. Simon (ed.), Elsevier Science Publishers BV, pp. 61-71, Amsterdam, 1989.

[6] F. Meyer and J.Serra, "Contrast and activity lattice", Signal Processing 16 (4), 303-317 (1989).

[7] S. Velasco-Forero, J. Angulo, and P. Soille, "Conditional toggle mappings: principles and applications", J. Mathematical Imaging and Vision 48, 544-565 (2014).

[8] J. Angulo, "Morphological colour operators in totally ordered lattices based on distances: application to image filtering, Enhancement and Analysis", Computer Vision and Image Understanding 107, 56-73 (2007).

[9] S. Skoneczny, "Nonlinear image sharpening in the HSV color space", Przeglad Elektrotechniczny (Electrotechnical Review) 88 (2), 140-144 (2012).

[10] P. Soille, "A note on morphological contrast enhancement", Technical Report RT-PS-001 Ecole de Mines d'Ales-EERIE, (1997).

[11] P. Soille, Morphological Image Analysis, Springer-Verlag, Berlin, 2003.
[12] A. Evans, "Nonlinear operations for colour images based on pairwise vector ordering", Proc. Digital Image Computing: Techniques and Applications - DICTA 1, 61-65 (2005).

[13] V. Barnett, "The ordering of multivariate data", J. Royal Statistical Society 139 (3), 318-355 (1976).

[14] M. Comer and E. Delp, "Morphological operations for color image processing", J. Electron. Imaging. 8 (3), 279-289 (1999).

[15] E. Aptoula and S. Lefevre, "A Comparative study on multivariate mathematical morphology", Pattern Recogn. 40, 2914 2929 (2007).

[16] C.-J. Zhao, X.-H. Gao, and X.-Y. Hu, "An adaptive lexicographical ordering of color mathematical morphology", J. Comp. 24 (1), 51-59 (2013).

[17] S. Velasco-Forero and J. Angulo, "Vector ordering and, multispectral morphological image processing", in Advances in Low-Level Color Image Processing, eds. M. Enre Celebi, B. Smolka, pp. 223-239, Berlin, 2014.

[18] E. Aptoula and S. Lefevre, "On lexicographic ordering in multivariate mathematical morphological”, Pattern Recogn. Let. 29 (2), 109-118 (2008).

[19] F. Ortiz, F. Torres, J. Angulo, and S. Puente, "Comparativ6e study of vectorial morphological operations in different color spaces", Proc. SPIE 4572, 259-268 (2001).

[20] M. Vardavoulia, I. Andreadis, and Ph. Tsalides, "Vector Ordering and morphological operations for colour image processing: fundamentals and applications", Pattern Analysis Applications 5, 271-287 (2002).

[21] A. Dhawan, G. Buelloni, and R. Gordon, "Enhancement of mammographic features by optimal adaptive neighborhood image processing", IEEE Trans. Med. Imaging. 5 (1), 8-15 (1986).

[22] S. Matz and R. de Figueiredo, "A Nonlinear image contrast sharpening approach based on Munsells scale", IEEE Trans. Imag. Proc. 15, 900-909 (2006). 